\title{
Atheroembolic renal disease and rivaroxaban: A new association
}

\author{
Catarina Isabel Ribeiro ${ }^{1}$, João Pedro Abreu ${ }^{2}$, Clara Almeida ${ }^{1}$, Ana Ventura ${ }^{1}$, Clara Santos ${ }^{1}$, Cátia Cunha ${ }^{1}$, David Tente ${ }^{3}$, Agostinho Sanches ${ }^{3}$, João Carlos Fernandes ${ }^{1}$ \\ ${ }^{1}$ Serviço de Nefrologia - Centro Hospitalar de Vila Nova de Gaia/ Espinho \\ 2 Serviço de Medicina Interna - Hospital de Santa Maria Maior - Barcelos \\ ${ }^{3}$ Serviço de Anatomia Patológica - Centro Hospitalar de Vila Nova de Gaia/ Espinho
}

\section{ABSTRACT}

Atheroembolic renal disease is a multisystem clinical entity of unknown incidence. It is often associated with severe atherosclerotic disease and its etiology can be spontaneous or iatrogenic. Its pathophysiology is based on renal dysfunction secondary to embolization of cholesterol crystals, followed by occlusive phenomena affecting the renal vasculature. The present report describes the case of a patient with several cardiovascular risk factors, with a recent introduction of rivaroxaban. The clinical course presented was suggestive of renal atheroembolic disease, and the histological evaluation corroborated this diagnosis.

Keywords: Acute Kidney Injury, Atheroembolic renal disease, Rivaroxaban.

\section{INTRODUCTION}

Currently, and assuming a multisystemic character, it is recognized that the embolization of cholesterol crystals can affect the kidney, the skin, the gastro-intestinal tract and the brain. ${ }^{1}$

Atheroembolic renal disease is a secondary form of renal injury that consists of embolization of cholesterol crystals into the distal vasculature, with partial or total occlusion of small arteries, arterioles and glomerular capillaries. ${ }^{1,2}$ The material coming from the atheromatous plates moves after their rupture. This process can be spontaneous or triggered by intravascular manipulation or by the administration of drugs (fibrinolytics and anticoagulants).

In about $70 \%$ of cases, the triggering iatrogenic event is identified, with endovascular procedures standing out as the most common trigger (80\%). The start of hypocoagulation is described in about $7 \%$ of all iatrogenic episodes, and while evidence in the literature for this association is scarce, it is increasing. 1,3

\section{CASE REPORT}

The authors present the case of a male patient, 75-years-old and Caucasian, with no known previous kidney disease or diabetes mellitus. He was smoker and had a known history of arterial hypertension, dyslipidemia, ischemic heart and cerebrovascular disease. The patient had a low risk and idiopathic pulmonary thromboembolism, and had been hypocoagulated with rivaroxaban since this event.

Seven months after this thromboembolic episode, the patient was admitted to Nephrology Service for a work-up due to a constitutional picture and acute kidney injury. The anamnesis referred to asthenia, anorexia and unquantified weight loss, with more than three months of evolution. There was no mention of other symptoms. On physical examination on hospital admission, he was hypertensive (blood pressure $180 / 102 \mathrm{mmHg}$ ), normocardial (heart rate 67 beats/minute), apyretic (temperature $36.3^{\circ} \mathrm{C}$ ), eupneic (respiratory rate $12 / \mathrm{min}$ ) and without pain. He was euvolemic and with preserved diuresis. There were no objective cutaneous and mucous changes and no signs of peripheral hypoperfusion. The requested analytical study documented an elevation serum creatinine $(4.80 \mathrm{mg} / \mathrm{dL}$ ), hyperglycemia $(230 \mathrm{mg} /$ $\mathrm{dL}$ ) with $\mathrm{HbA1c} 7.1 \%$, normochromic and normocytic anemia with hemoglobin $9.0 \mathrm{~g} / \mathrm{dL}$, erythrocyte sedimentation velocity $120 \mathrm{~mm} / \mathrm{H}$ and $\mathrm{C}$ reactive protein $2.60 \mathrm{mg} / \mathrm{dL}$. There was no leukocytosis or peripheral eosinophilia. The urine exam showed sediment without leuko-erythrocyturia or eosinophiluria, and the protein-creatinine ratio in an occasional sample was 0.50 . Renal tract ultrasonography presented normal kidney dimensions, with regular contours and preserved parenchymal thickness. There was no signs of obstructive uropathy or organized liquid collections or expansive lesions. The immunological study including the serum anti-nuclear factor assay and the anti-neutrophil cytoplasm antibody was negative; the serum complement (fraction C3 and C4), the protein electrophoresis and serum and urine immunofixation were normal. Serology test for human immunodeficiency virus and $B$ and $C$ hepatitis were negative.

The patient was submitted to percutaneous kidney biopsy (Figure 1 and Figure 2). Histology by light microscopy showed a biconvex slit-like cholesterol crystal in a medium caliber artery, with cellular inflammatory reaction and the presence of some macrophages. Arteriole hyalinosis was visible. Glomerular basal membrane thickening and mesangial expansion was also present with interstitial fibrosis, lymphocytic infiltration, and tubular atrophy. Immunofluorescence revealed linear IgG deposit and albumin in the glomerular basement membrane. Electron microscopy wasn't performed. 


\section{Figure 1}

Periodic Acid-Schiff staining shows a biconvex slit-like cholesterol crystal in a medium caliber artery, with cellular inflammatory reaction and the presence of some macrophages (100X)

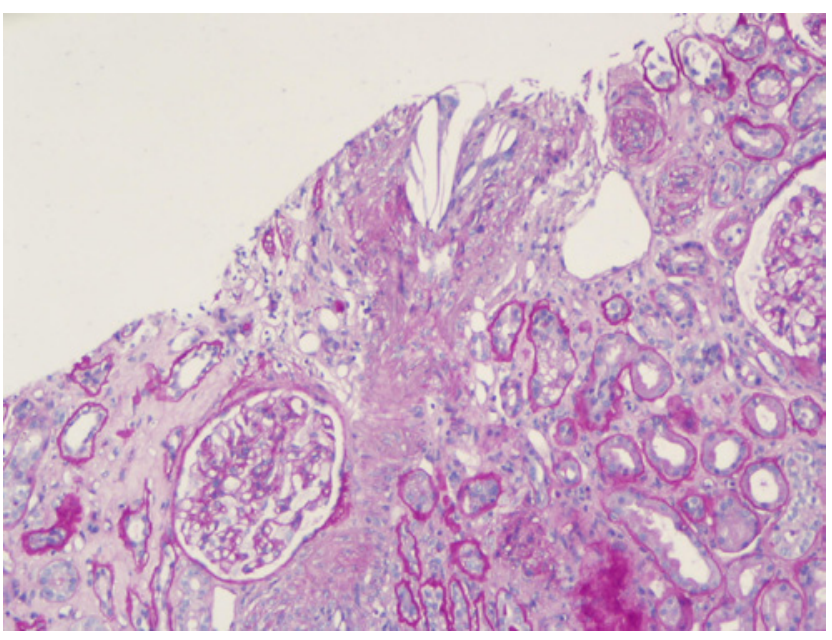

\section{Figure 2}

Masson trichrome stain shows large cholesterol cleft within arteriole lumen, with some interstitial fibrosis and tubular atrophy (200X)

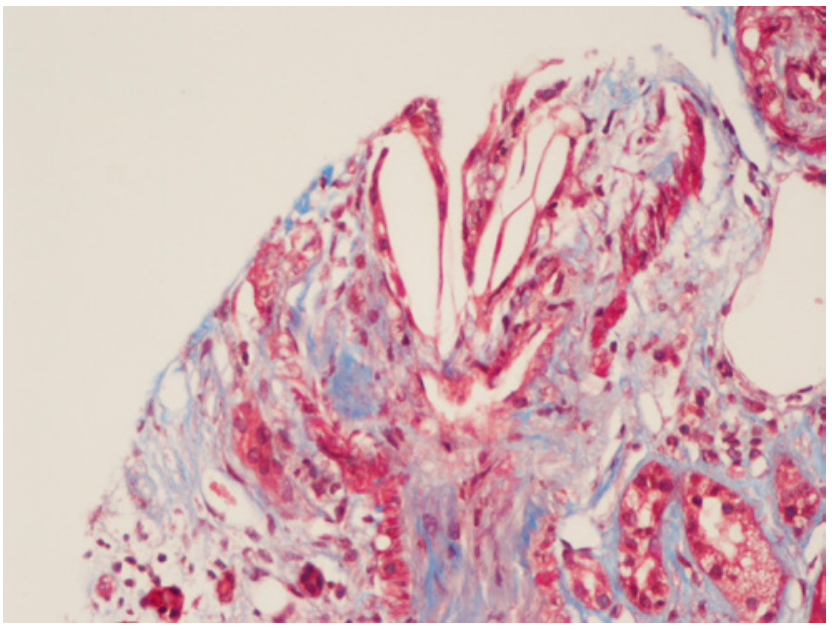

Histological integration with clinical and laboratory information confirmed the diagnosis of atheroembolic renal disease, with the most likely trigger being the start of hypocoagulation, seven months earlier.

The patient discontinued hypocoagulation with rivaroxaban and optimized therapy for the control of several cardiovascular risk factors. Another hypotensive was associated and an oral antidiabetic drug, an HMG co-A reductase inhibitor and an antiplatelet aggregator agent were initiated. However, the patient evolved with progressive deterioration of renal function, with severe azotemia and with no evidence of recovery. He needed to start dialysis therapy in hospital, with subsequent integration in a chronic hemodialysis program.

\section{DISCUSSION AND CONCLUSION}

Atheroembolic renal disease, due to its nonspecific clinical presentation, is an under-recognized pathology. ${ }^{1,2}$ Its diagnosis is dependent on a high degree of clinical suspicion, with a focus on a detailed anamnesis and physical examination. In the coming years, its incidence is expected to increase, not only due to the greater longevity of patients with diffuse atherosclerosis, but also to a greater number of invasive vascular procedures and the widespread use of anticoagulants and fibrinolytics in clinical practice. ${ }^{2,3}$

Atheroembolic renal disease has a classic triad that combines the presence of acute or subacute renal injury with signs of peripheral embolization and exposure to a triggering event. When present, this triad establishes the diagnosis itself, eliminating the need for additional histological evaluation. ${ }^{1,2,5}$ In the present case, there was no sign of involvement of other organs, with only nonspecific constitutional symptoms of asthenia, anorexia and unquantified weight loss, without objectifying the typical findings of peripheral involvement. Given the absence of vascular manipulation and assuming the scarcity of bibliography on atheroembolic renal disease associated with new oral anticoagulants (including rivaroxabano), percutaneous kidney biopsy proved to be essential.

In atheroembolic renal disease, and similarly to what was seen in the present case, histological findings illustrate the occlusion of small and medium-sized arteries by cholesterol crystals from atherosclerotic plaques. ${ }^{5,6}$ Once constituted, these crystals indicate a cellular inflammatory reaction, with diffuse endothelial proliferation and subsequent fibrosis. Needle-shaped cholesterol crystals within the vessel lumen are pathognomonic and, as the present case, it permits the diagnosis of atheroembolic renal disease, distinguishing this from nephroangiosclerosis or other pathologies. ${ }^{7}$ The arteriole hyalinosis, the glomerular basal membrane thickening and the presence of linear IgG deposit and albumin in the immunofluorescence lead to the suspicion of a diagnosis of diabetic kidney disease. In spite of there being no previous history known of diabetes mellitus, there are registers of hyperglycemia with $\mathrm{HbA} 1 \mathrm{c}$ upper $6.5 \%$ at hospital admission.

Once there is no specific treatment, therapy should focus on the intensive control of cardiovascular risk factors and, whenever possible, avoidance of potential precipitating events. HMG co-A reductase inhibitors appear to be one of the most beneficial drugs in primary prevention, probably due to their stabilizing role in atherosclerotic plaques through lipid control and anti-inflammatory effect. The use of antiplatelet agents is controversial and strongly dependent on the need for secondary prevention. ${ }^{4,6}$

The poor prognosis of this disease reflects the severity of the underlying vascular pathology. Approximately $30 \%$ of patients, as in the present case, go on to need renal function replacement therapy. ${ }^{3}$

Cholesterol crystal embolisms have been described in association with the use of various anti-thrombotic drugs, and this complication 
can occur several weeks after the start of these drugs. ${ }^{8}$ In spite of the fact that cholesterol crystal embolisms can also be spontaneous, in the present case the authors consider that a coincident (and non-relational) effect with the start of the drug cannot be excluded.

The association of atheroembolic renal disease with the start of hypocoagulation is currently described in about $7 \%$ of iatrogenic reports. ${ }^{1,2}$ The contribution of new oral anticoagulants, probably due to their more recent use, isn't yet fully defined; the hypothesis of a lower risk of kidney injury associated with these has been raised when compared with vitamin $\mathrm{K}$ antagonists. However further studies are needed. ${ }^{9}$

In 2018, Muller-Hansma et al. presented two reports of cholesterol crystal embolisms associated with the use of an oral anticoagulant, received by Netherlands Pharmacovigilance Centre Lareb. One of these concerned an 80-year-old male, with medical history of aortic graft surgery, myocardial infarction treated with coronary angioplasty, multiple transient ischemic attacks and atrophy of the left kidney, who presented an atheroembolic renal disease about one week after starting oral anticoagulant (initially treatment with dabigatran and after 5 days replacement by edoxaban) for atrial fibrillation. The second case concerned a 71-year-old male with many cardiovascular risk factors, with diagnosis of atheroembolic renal disease about 5 weeks after administration of rivaroxaban for atrial fibrillation. ${ }^{10}$

The authors underline that although the time after initiation of the drug (7 months) described in the present case is not the most typical interval, a possible relationship exists between rivaroxaban and the diagnosis of atheroembolic renal disease. They emphasize that to date there are few described cases of this renal disease associated with hypocoagulation with rivaroxaban, justifying the relevance of this report.

Disclosure of potential conflicts of interest: none declared

\section{References:}

1. Scolari F, Ravani P. Atheroembolic renal disease. Lancet 2010; 375:1650.

2. Modi S, Rao K. Atheroembolic renal disease. J Am Soc Nephrol 2001; 12:1781.

3. Scolari F, Ravani P, Gaggi R, Santostefano M, Rollino C, Stabellini N, et al. The challenge of diagnosing atheroembolic renal disease: Clinical features and prognostic factors. Circulation 2007; 116:298.

4. Theriault J, Agharazzi M, Dumont M, Pichette V, Ouimet D, Leblanc M. Atheroembolic renal failure requiring dialysis: Potential for renal recovery? A review of 43 cases. Nephron Clin Pract 2003; 94:c11-8.

5. Hara S, Asada Y, Fujimoto S. Ateroembolic renal disease: Clinical findings of 11 cases. J Atheroscler Thromb 2002; 9:288-91.

6. Mann J, Thomas A. Treatment of atheroembolization with corticosteroids. Am J Hypertens. 2001;14:831-834

7. Antonucci F, Pizzolitto S, Travaglini M, et al. Atheroembolic renal disease: clinico-pathologic correlations. Adv Exp Med Biol. 1989;252:59-64.

8. Adya KA, Inamadar AC, Palit A. Anticoagulantes em dermatologia. Indian J Dermatol Venereol Leprol 2016; 82:626-40.

9. Munawar T, Ibe U, Jiwa N, Raissi S. Renal cholesterol crystal embolism in the setting of warfarin use. BMJ Case Rep 2019; 12(8).

10. Muller-Hansma AHG, Daemen-Gubbels CRGM, Schut NH. Cholesterol embolisms as possible adverse drug reaction of direct oral anticoagulants. Neth J Med 2018; 76(3):125-8.

\section{Correspondence to:}

Catarina Isabel Correia Dias Ribeiro (https://orcid.org/0000-0001-6813-0336) Centro Hospitalar de Vila Nova de Gaia/ Espinho - Unidade I

Rua Conceição Fernandes, s/n | 4434-502 Vila Nova de Gaia, Porto - Portugal E-mail: catarina.isabel.ribeiro@gmail.com 